\title{
APLICAÇÃO DE UM MODELO DO DESEMPENHO AMBIENTAL INDUSTRIAL
}

\section{APPLYING A MODEL OF ENVIRONMENTAL PERFORMANCE INDUSTRIAL}

\author{
Camila Souza de Andrade ${ }^{1}$, Claudio Arcanjo de Sousa ${ }^{2}$, Fabiana Raupp ${ }^{3}$ \\ ${ }^{1}$ Universidade Federal da Grande Dourados UFGD - Dourados - Mato Grosso do \\ camilasouza.abdrade7@gmail.com \\ ${ }^{2}$ Faculdade de Ciências Biológicas e Ambientais - FCBA - Dourados - Mato Grosso do Sul; \\ arcanjo@ufgd.edu.br \\ ${ }^{3}$ Universidade Federal da Grande Dourados - UFGD - Dourados/ Mato Grosso do Sul; \\ fabianaraupp@ufgd.edu.br
}

\begin{abstract}
Resumo
Este artigo apresenta e discute a avaliação da aplicação do modelo de desempenho ambiental industrial, criado a partir da junção da norma ISO 14.031 (2004) e o método FMEA (Failure Mode and Effects Analysis). Para o desenvolvimento do mesmo, utilizou-se o levantamento dos aspectos e quantificação dos impactos ambientais, mensurando o risco ambiental de cada atividade, produto $e$ serviço exercido por um determinado empreendimento. $O$ método de pesquisa utilizado foi o estudo caso, aplicando o modelo em uma indústria de embalagens. Este modelo propõem indicadores de desempenho ambiental, denominados IDRA (Indicadores de desempenho e risco ambiental) que são capazes de diagnosticar possíveis falhas e melhorias no processo industrial, considerando que para o desenvolvimento do IDRA foram estabelecidos parâmetros de desempenho relativos a legislações aplicáveis, boas práticas adotadas pelo empreendimento, acordos e parcerias com terceiros e verificação de demandas mercadológicas. A partir dos resultados adquiridos nas avaliações dos parâmetros ambientais, formulou-se o calculo do IDRA determinando o desempenho ambiental da instituição, definindo os principais impactos a serem mitigados e as principais ações corretivas a serem tomadas. Após, a determinação do modelo de avaliação de desempenho ambiental industrial $e$ a aplicação deste na indústria de embalagens flexíveis foi obtido o valor de 61,3\% de desempenho ambiental, demonstrando que o empreendimento controla parte das interações da atividade empresarial com meio ambiente, porém apresenta diversas oportunidades de melhorias, $e$ ainda existem riscos a serem reduzidos e impactos a serem mitigados.
\end{abstract}

Palavras-chave: Gestão ambiental, avaliação de desempenho, indicadores de desempenho.

\section{Introdução}

A criação de modelos que utilizam métodos qualitativos para avaliar o desempenho ambiental vem sendo amplamente empregados em pesquisas, gestão e na tomada de 
decisão. Estabelecendo nossa confiança nas saídas de tais modelos é crucial para justificar o seu uso continuado ao mesmo tempo reconhecendo suas limitações, afim, de avaliar as caracteristicas de um modelo de desempenho em relação à compreensão e observações do seu sistema, resultando em diferentes abordagens e também na identificação de uma técnica mais apropriada (BENETT et al., 2013, p.2).

O desempenho ambiental pode ser medido através uma série de fatores e construtos que interagindo entre si permitam uma rápida visualização do comportamento e impacto dos indicadores ambientais dentro de um índice, que represente o seu desempenho. Neste campo encontram-se diversos modelos de medição dos resultados ambientais, mas que não deixam claro sua adequabilidade às diferenças existentes entre os meios onde as empresas estão situadas, variações estas, que dependem da localização geográfica, da existência de comunidades vizinhas, dos níveis de poluição aceitos pelos órgãos ambientais e outros (MOREL et al, 2004,p.3).

Segundo Ramos et al (2009,p.2) a avaliação de desempenho ambiental pode refletir por diferentes contextos, sendo uma ferramenta de gestão relacionada a tendência de melhoria ambiental, enfatizando sua eficiência, que proporciona analisar o estado do ambiente, onde faça cumprir as normas e regulamentos relacionados aos seus aspectos ambientais.

Assim, existe uma estreita relação entre o desempenho ambiental e gestão ambiental, uma vez que a gestão ambiental é um meio de alcançar o objetivo de melhorar desempenho ambiental. Assim, enquanto a gestão ambiental representa a aplicação de medidas de proteção ao ambiente, o desempenho ambiental tem como objetivo medir o grau de sua aplicabilidade através de métricas capazes de seguir seus objetivos ambientais (OrejaRodríguez; Armas-Cruz, 2012, p.2).

Neste contexto, é fundamental que as organizações adquiram ferramentas de gestão hábeis, que sejam capazes de propor estratégias ambientais que contemplem as necessidades legais, a eficiência no processo produtivo e o aprimoramento no planejamento ambiental. $\mathrm{O}$ modelo de avaliação de desempenho ambiental industrial faz a interligação entre o planejamento ambiental, avaliação dos aspectos e impactos, classificação do risco ambiental e o cumprimento das condicionantes legais, considerando que estes itens são importantes para a tomada de decisão. Este modelo é construído a partir de indicadores ambientais capazes de fornecer informações necessárias sobre o desempenho de uma determinada organização, fazendo com o que o mesmo consiga atingir seus objetivos e metas ambientais, almejando melhorias relacionadas ao processo produtivo.

O objetivo deste trabalho é aplicar o modelo de determinação do Índice de Desempenho Ambiental industrial em uma indústria de embalagens no município de Dourados, MS.

\section{Materiais e Métodos}

\subsection{Pesquisa: Modelo de avaliação de desempenho ambiental industrial}


Há várias formas de medir o desempenho e o risco ambiental nas organizações. Haja vista, que algumas metodologias não deixam claro seus objetivos e resultados. A NBR ISO 14.031 (2004) contribui como ferramenta para o auxilio na medição de desempenho ambiental, possibilitando também a criação de índices de riscos ambientais através da junção da metodologia FMEA. O uso destes métodos em conjunto é fundamental para adequação da gestão relacionado aos aspectos e impactos ambientais significativos.

O presente trabalho foi desenvolvido através de um modelo de avaliação de desempenho e risco ambiental, baseados nos conceitos adotados pela NBR ISO 14.031 e o método FMEA.

Este modelo é construído pela interligação entre estas duas metodologias. Os conceitos da NBR ISO 14.031 são para dar suporte ao gerenciamento interno e ao desenvolvendo indicadores ambientais capazes de auxiliar na tomada de decisão. $\mathrm{O}$ indicador empregado neste modelo é o IDRA (Indicador de Desempenho e Risco Ambiental) cuja função é levantar os aspectos e impactos ambientais relacionados às atividades, processos, produtos e serviços da organização. O método FMEA junta-se ao IDRA para determinar a quantificação dos impactos ambientais provenientes de cada atividade, serviço e produto realizado pelo empreendimento auxiliando no diagnostico ambiental e na classificação dos riscos ambientais.

\subsubsection{Aplicação do modelo}

O método de pesquisa adotado neste trabalho foi à modelagem qualitativa, a fim de construir um modelo e testa-lo em uma aplicação real. Para validação dos dados, o mesmo foi aplicado em uma indústria de embalagens flexíveis, localizada na cidade de Dourados-MS.

São premissas do modelo: (i) levantamento dos aspectos ambientais significativos; (ii) quantificação dos graus de impacto; (iii) determinação dos parâmetros de desempenho; (iv) quantificação do grau de desempenho; (v) interpretação dos resultados finais.

\subsubsection{Levantamento dos aspectos ambientais}

A primeira etapa para o desenvolvimento deste modelo fez-se a partir do levantamento de todos os aspectos das atividades, processos, produtos e serviços do empreendimento. Nesta etapa foi utilizado um check list para a seleção de dados qualitativos de cada setor, analisando as etapas do processo industrial com suas entradas e saídas correspondentes a cada etapa do processo, o Check List foi realizado com diretor industrial, gerente de produção e supervisores de cada setor analisado.

Após o preenchimento do Check List selecionou-se os aspectos ambientais significativos que compreenderam o gerenciamento de resíduos sólidos, uso dos recursos naturais e energéticos, emissões atmosféricas emitidas pelo empreendimento, dentre outros. 
Após a seleção dos principais aspectos ambientais, foi desenvolvida uma matriz contendo informações relevantes sobre a temporalidade, regime, incidência, classificação do aspecto e possíveis danos que trata os mesmos, conforme o Quadro 1.

Quadro 1 - Modelo de tabela da matriz de aspectos ambientais

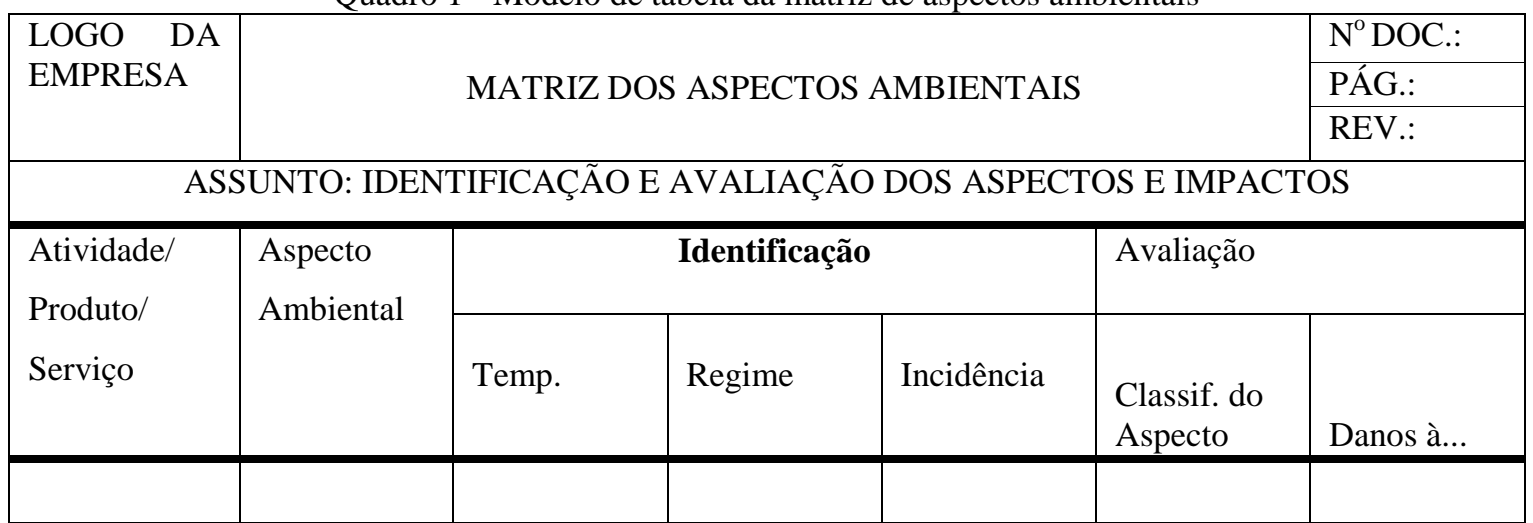

Legenda:Temp.: Temporalidade; Classif. do aspecto: Classificação do Aspecto.

Fonte: Adaptado de Andrade; Turrioni (2000)

O Quadro 1 apresenta um exemplo da matriz de aspectos ambientais utilizada para a classificação de cada atividade e qual tipo de aspecto ambiental tal atividade gera ou poderá gerar de impacto ambiental, onde os campos foram preenchidos conforme os requisitos abaixo:

I. Atividade/Produto/Serviço: Inserir a atividade, produto ou serviço realizado pelo empreendimento;

II. Aspecto Ambiental: Inserir o tipo de aspecto ambiental que possa ser gerado através de determinada atividade/produto/ serviço realizado pelo empreendimento;

III. Identificação da temporalidade: Elemento resultante na ocorrência da temporalidade do aspecto, sendo ele classificado em atual, futuro ou passado;

IV. Identificação do regime: Resultante na identificação do regime de ocorrência deste aspecto, classificado em normal, anormal ou emergencial;

V. Identificação da Incidência: Resultante na incidência do aspecto, podendo ser classificado em direta ou indireta;

VI. Avaliação Classificação do Aspecto: Elemento que consiste na classificação do aspecto em serviço, atividade ou produto;

VII. Avaliação de Danos: Classificação do dano que o aspecto poderia causar, entre o homem, meio ambiente e instalações.

Cabe ressaltar que com a elaboração da matriz de aspectos ambientais permitirá identificar os impactos ambientais decorrentes das atividades/ e ou produtos encontrados no empreendimento, possibilitando mensurar seu grau de impacto ambiental.

\subsubsection{Mensuração dos impactos ambientais}


A mensuração dos impactos ambientais foi determinada utilizando a ferramenta FMEA, adaptada ao diagnóstico e verificação dos riscos ambientais, onde sua formulação foi realizada a partir dos dados da fase do levantamento dos aspectos ambientais, utilizando a classificação das principais atividades/serviço/ produto e seus respectivos aspectos. Quadro 2.

\begin{tabular}{|c|c|c|c|c|c|c|}
\hline \multirow{3}{*}{$\begin{array}{l}\text { LOGO DA } \\
\text { EMPRESA }\end{array}$} & \multirow{3}{*}{\multicolumn{5}{|c|}{$\begin{array}{c}\text { MODELO FMEA ADAPTADA AO DIAGNOSTICO E VERIFICAÇÃO } \\
\text { DE RISCOS AMBIENTAIS }\end{array}$}} & $\mathrm{N}^{\mathrm{o}}$ DOC.: \\
\hline & & & & & & PÁG.: \\
\hline & & & & & & REV.: \\
\hline \multicolumn{7}{|c|}{ ASSUNTO: IDENTIFICAÇÃO E AVALIAÇÃO DOS IMPACTOS } \\
\hline $\begin{array}{l}\text { Impacto } \\
\text { ambiental }\end{array}$ & Severidade & Probab. & Detec. & Atividade & Produto & $\begin{array}{l}\text { Tipo } \\
\text { de risco }\end{array}$ \\
\hline & & & & & & \\
\hline
\end{tabular}

Legenda: Probab.: Probabilidade; Detec.: Detecção.

Fonte: Adaptado de Andrade; Turrioni (2000)

O Quadro 2 apresenta o modelo adaptado do FMEA utilizado para diagnostico e verificação dos riscos ambientais, os critérios adotados foram:

I. Impacto ambiental: Inserir o impacto ambiental correspondente ao aspecto ambiental relacionado à atividade/serviço/produto;

II. Severidade: Grau de severidade relacionada ao impacto causado ao meio ambiente;

III. Probabilidade: Consiste na probabilidade de incidência de ocorrer o impacto ambiental;

IV. Detecção: Consiste no grau de detecção do impacto gerado;

V. Atividade: Grau de atividade que irá gerar este impacto;

VI. Produto: Soma final de cada critério.

A classificação do risco ambiental é realizada através da multiplicação dos quatros parâmetros estabelecidos pelo FMEA (severidade, probabilidade, atividade e detecção) que variam entre valores de 1 á 5 . O valor final dado pela multiplicação destes parâmetros será a classificação do risco ambiental, conforme destaca a Tabela 1. Ressaltando que esta multiplicação servirá para calcular o grau de impacto de cada atividade, produto ou serviço que é desenvolvido pelo empreendimento.

Tabela 1 - Ponderação dos riscos ambientais

\begin{tabular}{cc}
\hline Criticidade & Tipo de Risco \\
\hline Até 9 & Riscos Triviais \\
Severidade menor que 3 & Riscos Toleráveis \\
De 10 a 24 & \\
Severidade menor ou igual a 3 & Riscos Moderados \\
De 25 a 84 & Riscos Relevantes \\
Severidade menor ou igual a 4 &
\end{tabular}


Fonte: Autoria própria (2013)

A classificação dos riscos ambientais foi composta pelo grau de criticidade de cada aspecto, e o mesmo foi classificado como triviais, toleráveis, moderados, relevantes e intoleráveis, conforme a Tabela 2.

Tabela 2 - Classificação dos riscos ambientais

\begin{tabular}{|c|c|}
\hline Tipo de Risco & Nível de Ação \\
\hline Riscos Triviais & Não necessitam de ações especiais, e nem preventivas. \\
\hline Riscos Toleráveis & $\begin{array}{l}\text { Não requerem ações imediatas, e poderão ser implementadas pela gerencia, } \\
\text { quando achar oportuno. }\end{array}$ \\
\hline Riscos Moderados & $\begin{array}{l}\text { Requer previsão e definição de prazo, cujo o prazo estipulado seja curto, indicar } \\
\text { responsáveis pela implementação das ações. }\end{array}$ \\
\hline Riscos Relevantes & $\begin{array}{l}\text { Exige implementação imediata das ações preventivas e de detecção, formulando } \\
\text { as responsabilidades especificas, com base no monitoramento contínuo. }\end{array}$ \\
\hline Riscos Intoleráveis & $\begin{array}{l}\text { Os trabalhos não poderão ser iniciados e deverão ser interrompidos de imediato, } \\
\text { somente poderão ser reiniciados após implementação de ações de corretivas e } \\
\text { monitoramento continuo. }\end{array}$ \\
\hline
\end{tabular}

Fonte: Autoria própria (2013)

Para a interpretação dos valores adotados pela matriz de aspectos e a mensuração de impactos com o uso do FMEA adotou-se parâmetros de desempenho que foram responsáveis para determinação e quantificação do índice de desempenho e risco ambiental.

\subsubsection{Aplicação dos parâmetros de desempenho}

Os parâmetros de desempenho aplicados neste trabalho foram:

a) Levantamento das legislações aplicáveis: foram levantadas todas as legislações ambientais de âmbito Federal, Estadual e Municipal, aplicáveis e a outros requisitos subscritos pela organização relacionados aos seus aspectos ambientais.

b) Levantamento das boas práticas adotadas pelo empreendimento: foram analisadas as ações e boas práticas adotadas pela organização que estejam relacionadas aos seus aspectos ambientais. Tais ações são realizadas de forma voluntárias, não havendo obrigações legais para as mesmas.

c) Verificação de acordos e parcerias: foram analisados os acordos e parcerias com terceiros que estivessem relacionadas aos aspectos ambientais identificados a partir da avaliação dos aspectos ambientais.

d) Verificação das demandas mercadológicas: foram levantadas as ações desenvolvidas pelo empreendimento que seja motivado por exigências de clientes e ou fornecedores.

\subsubsection{Quantificação do índice de desempenho ambiental}

A quantificação do índice de desempenho ambiental foi estabelecida a partir da determinação dos parâmetros de desempenho realizada através da aplicação de um questionário 
qualitativo com todos os aspectos identificados nas fases anteriores, este questionário foi aplicado a cada setor analisado. No caso específico deste empreendimento, foram identificadas sessenta e quatro questões com três opções de respostas: não atendimento ao item de controle do aspecto; atendimento parcial e atendimento total. O questionário foi dividido em sessões, compostas por perguntas relacionadas às suas atividades classificadas em:

i. Atendimento a Legislação com impacto legal e ambiental

ii. Atendimento a Legislação com impacto legal

iii. Mercado

iv. Política interna

v. Parcerias e acordos

vi. LAIA - Levantamento de aspectos e impactos ambientais

Cada sessão estabelecida no questionário do IDRA corresponde á aspectos diferenciados, relacionados diretamente com os parâmetros de desempenho proposto neste trabalho. A ponderação dos aspectos ambientais foi estabelecida a partir do aspecto que obteve o maior impacto ambiental (referência), como isso fez com que definisse as ponderações para os demais aspectos a serem considerados. Pelo grau de importância, o parâmetro atendimento a legislação teve o mesmo peso do maior impacto ambiental detectado pelas atividades do empreendimento. Tais ponderações estão apresentadas na Tabela 3.

Tabela 3 - Ponderação dos aspectos ambientais para formulação do IDRA

\begin{tabular}{lcc}
\hline \multicolumn{1}{c}{ Aspecto } & Peso & Percentual \\
\hline Atendimento a Legislação-impacto legal e ambiental & 10 & 1 \\
Atendimento a legislação-impacto legal & 8 & 0,8 \\
Mercado & 5 & 0,5 \\
Política interna & 3 & 0,3 \\
Parcerias e acordos & 3 & 0,3 \\
LAIA - Lev. Aspectos e Impactos ambientais & \multicolumn{2}{c}{ Padrão } \\
\hline
\end{tabular}

Fonte: Autoria própria (2013)

Para cada resposta de determinada ação, o empreendimento recebia uma pontuação que variava em função do atendimento aquela ação. Atendimento total representa $100 \%$ da pontuação máxima possível de se obter naquela ação, atendimento parcial, representa $50 \%$ da pontuação máxima possível de se obter naquela ação e não atendimento representa $0 \%$ da pontuação máxima possível de se obter naquela ação.

O cálculo do IDRA foi realizado através do somatório da pontuação obtida dividido pela pontuação máxima possível de se obter. A Figura 1 representa o calculo do IDRA. 


$$
\text { IDRA }=\frac{\sum \text { Pontos obtidos }}{\sum \text { Máximo a obter }} \times 100
$$

Figura 1 - Cálculo do IDRA. Fonte: Autoria própria (2013).

A partir da pontuação obtida do resultado do cálculo do IDRA será realizada a classificação do desempenho e risco ambiental do empreendimento, conforme a Tabela 4.

Tabela 4- Pontuação, conceito e descrição dos valores obtidos no IDRA

\begin{tabular}{lll}
\hline Pontuação & \multicolumn{1}{c}{ Conceito } & \multicolumn{1}{c}{ Descrição } \\
\hline $0-29 \%$ & ELEMENTAR & $\begin{array}{l}\text { O desempenho ambiental do empreendimento é elementar e } \\
\text { apresenta riscos elevados de autuação por parte dos órgãos } \\
\text { competentes. }\end{array}$ \\
$30-49 \%$ & INSATISFATÓRIO & $\begin{array}{l}\text { O desempenho ambiental do empreendimento é insatisfatório e } \\
\text { apresenta riscos moderados de autuação por parte dos órgãos } \\
\text { competentes, não garantindo segurança ao meio ambiente. }\end{array}$ \\
$50-69 \%$ & ADEQUADO & $\begin{array}{l}\text { O desempenho ambiental do empreendimento permite controlar } \\
\text { parte das interações da atividade empresarial com o meio } \\
\text { ambiente e também apresenta oportunidades de melhoria. }\end{array}$ \\
$70-89 \%$ & SATISFATÓRIO & $\begin{array}{l}\text { O empreendimento adota procedimentos adequados de gestão, } \\
\text { controle e prevenção dos impactos ambientais. }\end{array}$ \\
& EXCELENTE & $\begin{array}{l}\text { O empreendimento adota ações ou possui um sistema de gestão } \\
\text { ambiental que analisa, avalia e gerencia com eficácia os riscos } \\
\text { ambientais, possuindo um padrão de gestão ambiental que busca } \\
\text { a melhoria continua. }\end{array}$
\end{tabular}

Fonte: Autoria própria (2013)

\section{Resultados e discussão}

Os resultados preliminares foram a partir do levantamento dos aspectos ambientais significativos, onde foram divididos em setores, tais como administrativo, produção, limpeza e classificados por percentual decorrente sua incidência. Considerando que dentre as áreas estudadas, os percentuais mais relevantes sobre a ocorrência destes aspectos foram nos setores de armazenamento de resíduos e administração, onde ambos contribuíram com $26 \%$ do total dos resultados adquiridos, como pode ser observada na Tabela 4.

Tabela 4 - Análise dos aspectos de cada setor do empreendimento

\begin{tabular}{lcc}
\hline Atividade Setor Administrativo & Aspectos & \% \\
\hline Administração & 10 & 13,0 \\
Manutenção & 6 & 7,8 \\
Transporte & 6 & 7,8 \\
Serviços de Primeiro Socorros e Odontológico & 5 & 6,5 \\
Movimentação de Veículos & 2 & 2,6 \\
\hline Atividade Setor Produção & Aspectos & $\%$ \\
\hline \multicolumn{1}{c}{ Impressão de embalagens } & 8 & 10,4
\end{tabular}




\begin{tabular}{lcc}
\hline Atividade Setor Administrativo & Aspectos & $\%$ \\
\hline Armazenamento de Produtos & 3 & 3,9 \\
Acabamento do Produto & 2 & 2,6 \\
Análise Laboratorial & 2 & 2,6 \\
Extrusão & 2 & 2,6 \\
Laminação & 2 & 2,6 \\
Gestão de Aparas & 1 & 1,3 \\
Laboratório de Tintas & 1 & 1,3 \\
Qualidade do Processo & 1 & 1,3 \\
\hline Atividade Setor Limpeza & & 13,0 \\
\hline Armazenamento de Resíduos & 10 & 9,1 \\
Organização e Limpeza & 7 & 2,6 \\
Higiene Pessoal & 2 & 2,6 \\
Varrição e Jardinagem & 2 & $\mathbf{1 0 0 \%}$ \\
\hline Total & & \\
\hline Fonte: Autoria própria (2013) & \\
\hline
\end{tabular}

Fonte: Autoria própria (2013)

A partir dos resultados correspondentes aos dados levantados anteriormente, pode-se notar que dependendo da atividade realizada pelo empreendimento poderá ocorrer uma quantidade maior de aspectos ambientais, e consequentemente a maior incidência de impactos. Neste sentido, alguns aspectos ambientais obtiveram maior visibilidade, proporcionando uma análise ponderada sobre a ocorrência de seus impactos e o grau criticidade que o mesmo pode acarretar. Para esta análise utilizou a ferramenta de análise de modo de efeito e falha denominada de FMEA que quantificou cada impacto relacionado ao seu respectivo aspecto e seu grau de risco ambiental.

Os resultados do FMEA se deram a partir do levantamento dos aspectos ambientais onde foram qualificados os riscos ambientais que incidiam sob as atividades do empreendimento analisado. Neste estudo os riscos encontrados foram classificados como triviais, toleráveis, moderados e relevantes. No entanto, não foram encontrados riscos com grau de impacto intolerável. Este fato pode ser explicado pelo grau de impacto baixo da atividade e pelas medidas de controles adotadas para os mesmos, bem como pelo porte médio do empreendimento (Figura 2).

O uso do FMEA como ferramenta de gestão sendo está sendo um item essencial para avaliação dos aspectos e impactos ambientais, fazendo com que esta metodologia seja aprimorada. "Vários autores estão utilizando esta ferramenta como suporte a gestão ambiental (OLIVEIRA; FREITAS, 2013; KANIA et al., 2014; ROSZAK et al., 2014; SENNA, et al., 2014)". Neste trabalho o FMEA também foi utilizado como uma peça chave para o desenvolvimento de um modelo de gestão. 
Figura 2 - Classificação do risco ambiental

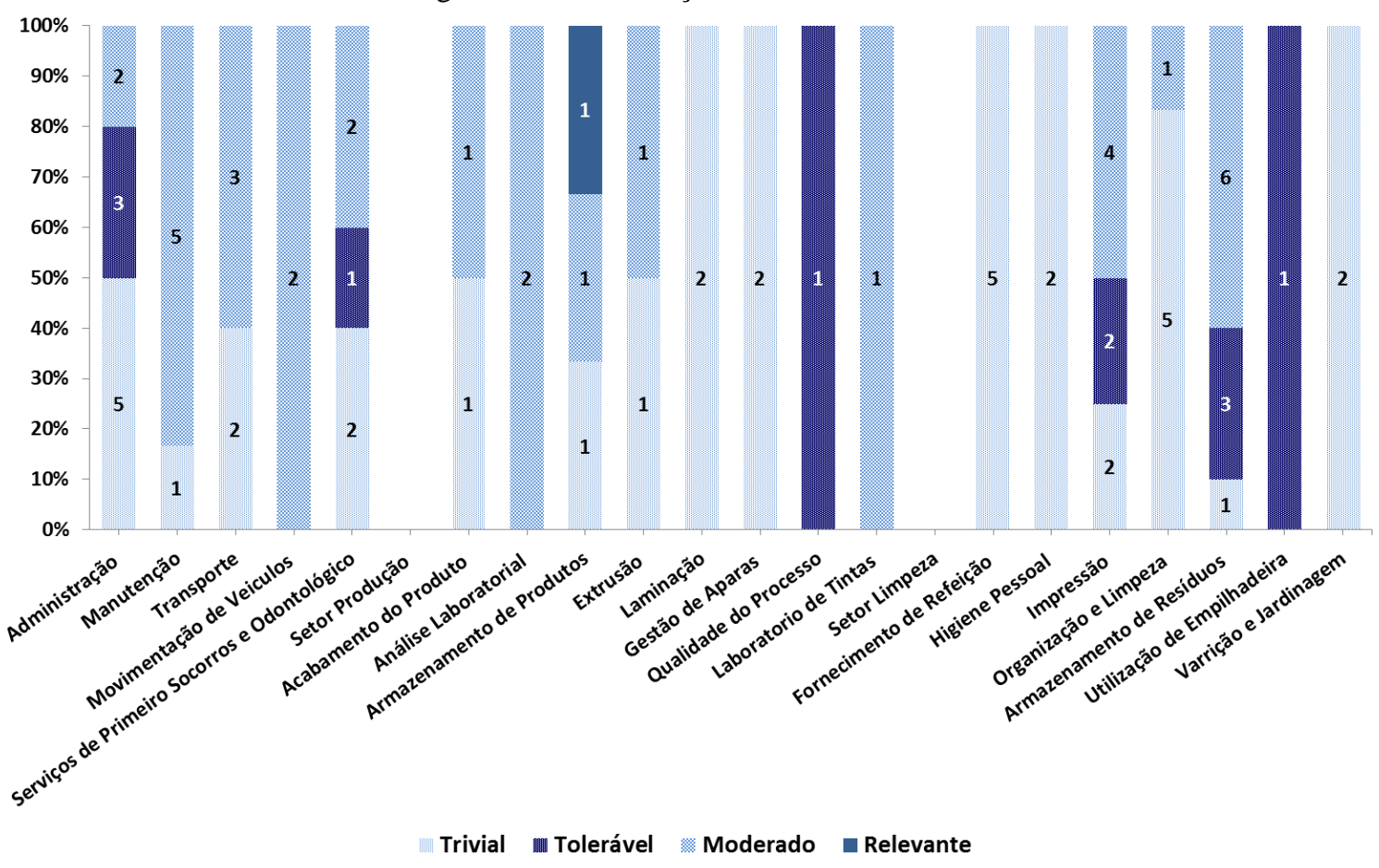

Fonte: Autoria própria (2013)

Pode-se observar que as atividades relacionadas ao armazenamento de produtos obteve em um dos seus aspectos a classificação de risco considerado relevante, sendo este classificado como um risco que pode ser prevenido e controlado. Embora a atividade de armazenamento de resíduo não tenha nenhum risco relevante ou intolerável, esta atividade apresentou a maior quantidade de risco moderado, determinando que a mesma requeira cuidado especial na operação de modo a evitar tais impactos ambientais.

Os riscos ambientais que obtiveram maior ocorrência foram os triviais, toleráveis, moderados e relevantes. Neste estudo não foi encontrado nenhum risco ambiental com grau de impacto intolerável. Este fato se explica pelo fato de que o empreendimento é de porte médio, suas atividades possuem baixo grau de impacto e o mesmo adota medidas de controles eficazes.

A partir da classificação dos riscos ambientais contidos na Figura 2 as atividades relacionadas ao armazenamento de produtos obteve em um dos seus aspectos a classificação de risco considerado relevante, sendo este classificado como um risco que pode ser prevenido e controlado. Embora a atividade de armazenamento de resíduo não tenha nenhum risco relevante ou intolerável, esta apresentou a maior quantidade de risco moderado, determinando que a mesma requeira cuidado especial na operação de modo a evitar tais impactos ambientais.

Por uma questão de critério de ponderação do modelo de avaliação do desempenho ambiental do empreendimento, foram descartados os impactos triviais e toleráveis, sendo utilizado somente os impactos moderados e relevantes. Os impactos moderados e relevantes estão apresentados no Quadro 1. 
Quadro 1. Representação das principais atividades que resultem em riscos ambientais

\begin{tabular}{|c|c|c|}
\hline Atividade & Aspecto & Impacto \\
\hline Administrativo & Descarte de pilhas, baterias entre outros & Contaminação do solo \\
\hline $\begin{array}{l}\text { Armazenamento de } \\
\text { Resíduos }\end{array}$ & $\begin{array}{l}\text { Descarte de resíduos contaminados classe I } \\
\text { (galões de tinta, solvente e óleo), descarte de } \\
\text { lâmpadas fluorescentes, consumo de } \\
\text { produtos agroquímicos. }\end{array}$ & $\begin{array}{l}\text { Perigo de contaminação e } \\
\text { vazamento de produtos químicos } \\
\text { perigosos e o r mau } \\
\text { acondicionamento de lâmpadas } \\
\text { fluorescentes podendo ocasionar } \\
\text { danos à saúde e meio ambiente. }\end{array}$ \\
\hline $\begin{array}{l}\text { Acabamento do } \\
\text { Produto }\end{array}$ & Geração de Ruído & $\begin{array}{l}\text { Ocasiona mal estar e perturbação } \\
\text { dos funcionários, } \\
\text { doenças do trabalhonondo }\end{array}$ \\
\hline $\begin{array}{l}\text { Análise } \\
\text { Laboratorial }\end{array}$ & Utilização de produtos químicos & $\begin{array}{l}\text { Podem ocorrer vazamentos e } \\
\text { contaminações. }\end{array}$ \\
\hline Impressão & $\begin{array}{l}\text { Volatilização de solvente/tinta e Efluentes } \\
\text { líquidos gerados pela lavagem das peças }\end{array}$ & $\begin{array}{l}\text { Ocasiona contaminação atmosférica } \\
\text { e contaminação do solo e água. }\end{array}$ \\
\hline $\begin{array}{l}\text { Laboratório } \\
\text { Tintas }\end{array}$ & $\begin{array}{l}\text { Armazenamento e descarte de latões com } \\
\text { borra de tinta }\end{array}$ & $\begin{array}{l}\text { Podem ocasionar vazamentos e } \\
\text { posteriormente contaminação do } \\
\text { solo e água }\end{array}$ \\
\hline Manutenção & Vazamento de óleo & $\begin{array}{l}\text { Pode ocorrer contaminação do solo } \\
\text { e água }\end{array}$ \\
\hline $\begin{array}{l}\text { Movimentação de } \\
\text { veiculo }\end{array}$ & Emissão de fumaça preta & Ocasionar emissão de $\mathrm{CO} 2$ \\
\hline $\begin{array}{l}\text { Serviços } \\
\text { Odontológicos e } \\
\text { Primeiros Socorros }\end{array}$ & $\begin{array}{l}\text { Descarte de resíduos classe I, resíduos classe } \\
\text { II A }\end{array}$ & $\begin{array}{l}\text { Ocasiona contaminação do solo e } \\
\text { água. }\end{array}$ \\
\hline $\begin{array}{l}\text { Armazenamento de } \\
\text { produto }\end{array}$ & Vazamento de produtos químicos & $\begin{array}{l}\text { Ocasiona contaminação do solo e } \\
\text { água. }\end{array}$ \\
\hline
\end{tabular}

Fonte: Autoria própria (2013)

O segundo resultado a ser obtido foi a ponderação de cada sessão contida no questionário do IDRA, possuindo como resultado final o desempenho ambiental do empreendimento, conforme desta a Tabela 7.

Tabela 7 - Índice desempenho ambiental do empreendimento.

\begin{tabular}{lcccc}
\hline Grupo de Aspectos Ambientais & $\begin{array}{c}\text { Valores } \\
\text { Máximos }\end{array}$ & $\begin{array}{c}\text { Máximo a } \\
\text { Obter }\end{array}$ & Obtido & IDA \\
\hline Atendimento a Legislação-Impacto Legal e Ambiental & 96,0 & 1248 & 1008 & $80,8 \%$ \\
\hline Atendimento a Legislação-Impacto Legal & 78,8 & 384 & 230 & $60,0 \%$ \\
\hline Mercado & 48,0 & 48 & 0 & $0,0 \%$ \\
\hline Política Interna & 28,8 & 893 & 447 & $50,1 \%$ \\
\hline Parcerias e Acordos & 28,8 & 58 & 29 & $50,3 \%$ \\
\hline LAIA - Lev. Aspectos e Impactos Ambientais (Padrão) & 96,0 & 396 & 140 & $35,4 \%$ \\
\hline Total & & & $\mathbf{6 1 , 3 \%}$ \\
\hline Fon:
\end{tabular}

Fonte: Autoria própria (2013) 
Após a formulação do modelo e aplicação do mesmo no empreendimento, observou-se o Índice de Desempenho Ambiental foi de 61,3\%. Seguindo as descrições contidas na Tabela 6, este percentual é considerado adequado, referindo-se que o empreendimento já possui um determinado controle sobre suas atividades e ações que possam intervir ao meio ambiente, porém apresenta oportunidades de melhoria decorrentes a algumas de suas atividades.

Como pode ser observado, o aspecto mercado foi o que obteve menor percentual, levando em consideração que a questão relacionada ao mesmo, era específica a mudança e inovação de mercado. Caso o empreendedor considerar que este não se aplica a sua atividade, o IDRA sofrerá um pequeno acréscimo em relação ao calculado, ficando o valor final de 62,2\%. O Levantamento dos Aspectos e quantificação dos Impactos Ambientais que foi referência para quantificação dos demais aspectos obteve $35,4 \%$, sendo considerado como o segundo menor valor a ser apresentado, ressaltando que vários aspectos que proviam de impactos significativos correspondidos a matriz do FMEA não são monitorados ou mitigados pelo empreendimento.

No entanto, o aspecto atendimento a legislação impacto legal e ambiental obteve maior valor perante as demais, haja vista que o empreendimento atende quase a totalidade de todas as exigências legais aplicáveis a atividade. Diante dos resultados adquiridos neste estudo, pode-se perceber que o empreendimento precisa de melhorias relacionadas à gestão ambiental. Considerando que muitos dos aspectos identificados em suas atividades, ainda não possuem parâmetros de prevenção e controle, comprometendo no resultado positivo do seu desempenho ambiental.

\section{Conclusão}

A aplicação do modelo de avaliação de desempenho ambiental industrial proporcionou ao empreendimento diagnosticar de forma abrangente seus aspectos ambientais, além de classificar o grau de criticidade de cada impacto auxiliando na melhor detecção dos riscos ambientais. Este modelo adequou-se aos padrões da ISO 14.031(2004) e do método FMEA pelo fato da necessidade de uma ferramenta capaz de mensurar e diagnosticar de forma simples o desempenho ambiental de um determinado empreendimento. Sendo assim, este trabalho seguiu todas as recomendações desta norma, onde a mesma sugere que seja feito levantamento de todos os aspectos ambientais (significativo e não significativo) e que os significativos sejam levados em consideração no estabelecimento, implementação e manutenção de seu sistema da gestão ambiental, ou seja, os impactos considerados triviais e toleráveis não entraram no computo geral do IDRA.

Em caso de implementação de ações para mitigação dos impactos ambientais moderados e relevantes, muitos dos impactos triviais e toleráveis serão comtemplados, exigindo assim uma nova avaliação. 
Este modelo possibilitou a criação de indicadores ambientais que poderão ser medidos periodicamente, fazendo com que o empreendimento estabeleça critérios para adoção do benchmarking interno e externo e definição de metas e implantação de planos de ações para adequar caso hajam desvios sendo considerado uma ferramenta eficaz para a gestão ambiental.

Conclui-se que desenvolvimento deste trabalho evidenciou a importância da utilização de metodologias de análise como FMEA e a determinação do Índice de Desempenho Ambiental, direcionadas a avaliação do desempenho ambiental, criando indicadores de desempenho que tenha por objetivo estabelecer ao empreendimento a visão geral do seu comportamento diagnosticando seus impactos. Cabe ressaltar que o índice de desempenho ambiental não é considerado um processo investigatório, mais sim um modelo comparado ao PDCA, objetivando o planejamento de suas ações, possibilitando atingir seus objetivos e metas que objetivem como resultado final o progresso ambiental.

\section{Abstract}

This article presents and discusses the evaluation of the implementation of industrial environmental performance model, created from the junction of ISO 14.031 (2004) and the FMEA (Failure Mode and Effects Analysis). For its development, we used the survey of aspects and quantification of environmental impacts, measuring the environmental risk of each activity, product and service exercised by a given enterprise. The research method used was the case study, applying the model in a packaging industry. This model proposed environmental performance indicators, called IDRA (performance indicators and environmental risk) that are able to diagnose possible failures and improvements in the industrial process, considering that for the development of IDRA were established performance parameters relating to applicable legislation, good practice adopted by the project, agreements and partnerships with third parties and verification market demands. From the results obtained in the assessments of environmental parameters, formulated to calculate the IDRA determining the environmental performance of the institution, setting out the main impacts to be mitigated and the main corrective actions to be taken. After the determination of the industrial environmental performance evaluation model and the application of the flexible packaging industry has achieved the value of $61.3 \%$ of environmental performance, demonstrating that the enterprise controls of the interactions of business activity with the environment, but It presents several opportunities for improvement, and there are still risks to be reduced and impacts to be mitigated.

Key-words: Environmental management, performance evaluation, performance indicators.

\section{Referências}

ANDRADE, M. R. S; TURRIONI, J.B. UMA METODOLOGIA DE ANÁLISE DOS ASPECTOS E IMPACTOS AMBIENTAIS ATRAVÉS DA UTILIZAÇÃO DO FMEA. Disponível em: $<$ http://www.ufrgs.br/sga/SGA/materialde-apoio/textos/textos

apoio/links/Uma\%20Metodologia\%20de\%20Analise\%20dos\%20Aspectos\%20e \%20Impactos\%20Ambientais\%20atrav es\%20da\%20Utilizacao\%20do\%20Fmea.pdf> Acesso: 01 de Julho de 2015.

ASSOCIAÇÃO BRASILEIRA DE NORMAS TECNICAS- NBR 14031- Gestão Ambeintal- Avaliação de Desempenho- Diretrizes, Rio de Janeiro: ABNT, 2004, 38 p.

BENNETT, N.D; CROKE N, B, F.W, GUARISO, G; GUILLAUME, J,H,A; HAMILTON, S, H; JAKEMAN, A,J; MARSILI-LIBELLI,S; NEWHAM, L,T,H.; NORTON, J, P; PERRIN, C; PIERCE, S, A; ROBSON, B; SEPPELT, R;. 
VOINOV, A, A;. FATH, B, D; ANDREASSIAN, V. Characterising Performance of Environmental Models. Environmental Modelling \& Software, v. 40 ,p.1-20, 2013. crossref

KANIA, A; Roszak, M; Spilka, M. Evaluation of FMEA methods used in the environmental management. Juornal Archives of Materials Science and Engineering. v. 65,N 1, p 37-44, 2014.

MOREL, P.E; LEÃO, T.G; SELLITO, A.M; CASTRO, O. S. Medição de desempenho ambiental em fabricação utilizando um método de apoio à decisão multicriterial. In. XXIV Encontro Nacional de Engenharia de Produção, Florianópolis, SC, 2004.

OLIVEIRA, L.N; FREITAS, L;S. O uso do FMEA como ferramenta de avaliação dos aspectos e impactos ambientais numa indústria de microeletrônica. Revista Gestão Industrial , v. 09, n04, p. 792-810, 2013.

OREJA-RODRÍGUEZ,J.R; ARMAS-CRUZ, Y. Environmental performance in the hotel sector: the case of the Western Canary Islands. Journal of Cleaner Production v. 29-30, p.64-72, 2012. crossref

RAMOS, T.B; ALVES, I; SUBTIL, R; MELO, J.J. The state of environmental performance evaluation in the public sector: the case of the Portuguese defence sector. Journal of Cleaner Production. v. 17. p 36-52, 2009. crossref

ROSZAK, M; SPILKA, M; KANIA, A. environmental failure mode and effects analysis (FMEA) - A new approach to methodology. Metalurgija, v 54, n 2,p 449-451, 2014.

SENNA, A.J.T; ALVES, R.R; SANTOS, N.R.Z; COSTA, F.L. Determinação do índice de risco ambiental das instalações de uma unidade de uma instituição federal multicampi de ensino superior. Revista Eletrônica em Gestão, Educação e Tecnologia Ambiental - REGET, v. 18 n. 1, p.555-565, 2014.

\section{Dados dos autores:}

Nome completo: Camila Souza de Andrade

Filiação institucional: Universidade Federal da Grande Dourados- UFGD

Departamento: Faculdade de Ciências Biológicas e Ambientais-FCBA

Função ou cargo ocupado: Estudante

Endereço completo para correspondência: Rodovia Dourados - Itahum, Km 12 - Cidade Universitária, Cx. Postal 533 - CEP 79804-97; Dourados, Mato Grosso do Sul

Telefones para contato: (67) 8122-4385

e-mail: camilasouza.andrade7@gmail.com

Nome completo: Claudio Arcanjo de Sousa

Filiação institucional: Universidade Federal da Grande Dourados- UFGD

Departamento: Faculdade de Ciências Biológicas e Ambientais- FCBA

Função ou cargo ocupado: Professor

Endereço completo para correspondência: Rodovia Dourados - Itahum, Km 12 - Cidade

Universitária, Cx. Postal 533 - CEP 79804-97; Dourados, Mato Grosso do Sul

Telefones para contato: (67) 9987-8709

e-mail:arcanjo@ufgd.edu.br

Nome completo: Fabiana Raupp 
Filiação institucional: Universidade Federal da Grande Dourados- UFGD

Departamento: Faculdade de Ciências Exatas e Tecnológicas- FACET

Função ou cargo ocupado: Professora

Endereço completo para correspondência: Rodovia Dourados - Itahum, Km 12 - Cidade

Universitária, Cx. Postal 533 - CEP 79804-97; Dourados, Mato Grosso do Sul

Telefones para contato: (67) 9696-2012

e-mail: fabianaraupp@ufgd.edu.br

Submissão: 09/08/2015

Aceito: $14 / 09 / 2015$ 